\title{
Über Dibenzylanthracen
}

von

\section{Eduard Lippmann und Isidor Pollak.}

Aus dem III. chemischen Universitätslaboratorium des Prof. E. Lippmann.

(Vorgelegt in der Sitzung am 15. Mai 1902.)

Wenn man Anthrachinon mit Zinkstaub und Lauge behandelt, so erhält man nach Liebermann ${ }^{1}$ Oxanthranol; wird dieses mit Alkylbromid und Lauge neuerdings gekocht, so entsteht das entsprechende Alkyloxanthranol, das, mit Alkohol und Salzsäure reduciert, Alkylanthracen liefert. Bach ${ }^{2}$ hat auf Veranlassung von Liebermann in dessen Laboratorium nach Liebermann's Methode das ganz analog constituierte Benzyloxanthranol und aus demselben mit Jodwasserstoff und Phosphor Monobenzylanthracen (S. 119) erhalten. In allen diesen Anthracenalkylderivaten ist das Alkyl an einen Kohlenstoff der Mittelstellung gebunden. Unsere Versuche, welche wir hier mittheilen wollen, bezwecken, vom Anthracen ausgehend, die Darstellung eines Dibenzylanthracens und seiner Derivate.

Darstellung. Nach einer Beobachtung, dass Anthracen in Schwefelkohlenstofflösung mit Benzylchlorid und etwas Zinkstaub unter Entwickelung von Chlorwasserstoff reagiert, haben wir $50 \mathrm{~g}$ Anthracen in der 20 fachen Schwefelkohlenstoffverdünnung mit der molecularen Menge Benzylchlorid, $75 \mathrm{~g}$, unter Zusatz von circa $1 \mathrm{~g}$ Zinkstaub bis zum Aufhören der Salzsäure-Entwickelung erhitzt, was circa 200 Stunden währte.

I Liebermann, Lieb. Ann., 212.

2 Berichte, 23, 1567 . 
Da eine Unterbrechung der Reaction hier nachtheilig wirkt, empfiehit es sich am besten, am constanten Wasserbade zu erwärmen. Kann man mit Ammoniak keine Saizsäure mehr nachweisen, so wird der Schwefelkohlenstoff abdestilliert und der Rückstand mit Äthe: aufgenommen. Hiebei geht eine Schmiere, die allen Bemühungen der weiteren Bearbeitung trotzte, in Lösung, während Krystalle von hellgelber Farbe zurückbleiben. Nachdem dieselben am Filter mit Äther sorgfältig ausgewaschen waren, wurden sie aus Benzol wiederholt umkrystallisiert. Ausbeute 40 bis $50 \%$ der Theorie. Man erhält schöne, weißlichgelbe, glänzende Nadeln vom Schmelzpunkte 239 bis $240^{\circ}$; thr specifisches Gewicht ist ein auffallend leichtes und beträgt bei $16^{\circ} 0 \cdot 1787$. Dieser Kohlenwasserstoff ist in Benzol, Schwefelkohlenstofi, Eisessig und Äther schwer löslich, hingegen in Alkohol und Ligroin unlöslich.

Analyse. $0.1500 \mathrm{~g}$ gaben $0.516 \mathrm{~g}$ Kohlensäure und $0.0840 \mathrm{~g}$ Wasser.

In 100 Theilen:

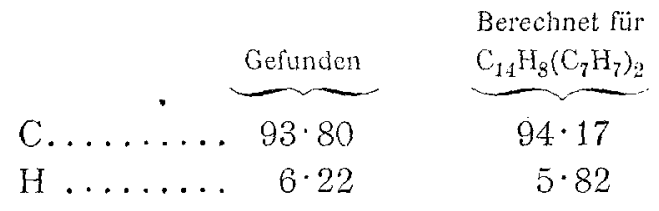

Da diese Analysenzahlen für das Monobenzylanthracen wie für das Dibenzylanthracen zutreffend sind, wäre es wünschenswert gewesen, das Moleculargewicht durch eine Moleculargewichtsbestimmung nach der Methode der Gefrierpunktserniedrigung oder Siedepunktserhöhung zu controlieren. Infolge der Schwerlöslichkeit der Substanz in den üblichen Lösungsmitteln, wie Benzol, Eisessig, Äther, musste vorläufig auf diese Methoden verzichtet werden.

Sind die beiden Benzylgruppen, wie anzunehmen war, an die beiden Kohlenstoffe in der Mesostellung gebunden, so mussten bei der Oxydation mit Chromsäure zwei Molectile Benzoesäure und ein Molecül Anthrachinon (58.1\%) entstehen.

Oxydation. $0.9638 \mathrm{~g}$ Dibenzylanthracen wurden in Eisessig gelöst, mit der entsprechenden Menge Chromsäure in der 
Kochhitze oxydiert, und zwar unter Zusatz von wenig Wasser. Die in der Kälte ausgeschiedenen Mengen Anthrachinon und Benzoesäure wurden abfiltriert, um mit jenen aus dem eingeengten Filtrat erhaltenen vereinigt zu werden. Der Filterrückstand wurde zur Entfernung der Benzoesäure mit Soda versetzt, in ein Becherglas gespült, mit Wasser gewaschen und auf ein bei $110^{\circ}$ getrocknetes Filter gebracht, nach dem Trocknen bis zur Gewichtsconstanz bei $110^{\circ}$ gewogen; die Gesammtmenge des auf diese Weise erhaltenen Anthrachinon betrug $0.5379 \mathrm{~g}$.

In 100 Theilen:

Anthrachinon $\underbrace{\text { Gefunden }}_{55 \cdot 8} \underbrace{\begin{array}{c}\text { Berechnet für } \\ \text { Dibenzylanthracen }\end{array}}_{58 \cdot 1}$

Da die Umsetzung eine fast quantitative, die Verluste infolge der Schwerlöslichkeit fast minimale sind, so begnügten wir uns mit dem Hinweise auf die Annäherung mit der gefundenen Zahl mit der für Dibenzylanthracen berechneten.

Das erhaltene Anthrachinon zeigte dén richtigen Schmelzpunkt von $273^{\circ}$, war in Eisessig sehr schwer löslich und erwies sich in allen sonstigen Eigenschaften mit dem aus Anthracen erhaltenen identisch; wären nun ein oder beide Benzylreste in den Kern getreten, dann hätte man bei der Oxydation ein Benzyl oder Dibenzylanthrachinon von abweichendem Schmelzpunkte und Eigenschaften erhalten sollen. Also würde dem Dibenzylanthracen nachstehende Structurformel zukommen:<smiles>CCCCCC12CC1(CCCC)C2CC</smiles> 
und die Oxydation würde nach folgender Gleichung erfolgen:

$$
\begin{aligned}
\mathrm{C}_{14} \mathrm{H}_{8}\left(\mathrm{CH}_{2} \mathrm{C}_{6} \mathrm{H}_{5}\right)_{2}+7 \mathrm{O}= & =\mathrm{H}_{2} \mathrm{O}+ \\
& +2 \mathrm{C}_{6} \mathrm{H}_{5}-\mathrm{COOH}+\mathrm{C}_{6} \mathrm{H}_{4} \mathrm{CO}^{\mathrm{CO}} \mathrm{C}_{6} \mathrm{H}_{4} .
\end{aligned}
$$

Bromierung, Bei besetzter Mesostellung war es naheliegend, dass das Bromatom in der Wärme einen Wasserstoff des $\mathrm{CH}_{2}$-Restes substituieren könnte, was durch nachstehende Versuche bestätigt wird. Moleculare Mengen Brom werden in Schwefelkohlenstofflösung, in moleculare Mengen Kohlenwasserstoff in demselben Mittel gelost, in der Kochhitze mittels Tropftrichter portionenweise eingetragen, bis die Entwickelung von Bromwasserstoff aufgehört und die braune Farbe der Flüssigkeit verschwunden ist. Nach Abdestillieren des Schwefelkohlenstoffes wurde der Rückstand behufs Entfernung des anhaftenden Bromwasserstoffes circa eine Stunde arm Wasserbade in einer Schale erwärmt; man erhält so ein gelbes krystallinisches Product, das, wie aus nachfolgenden Analysen hervorgeht, mit irgendeinem bromreicheren verunreinigt erscheint; zur weiteren Reinigung wurde es dreimal aus Schwefelkohlenstoff umkrystallisiert. Man erhält dann schöne, glänzende, gelbe Blättchen, welche unter Zersetzung bei $187^{\circ}$ schmelzen. Nach einmaligem Umkrystallisieren erhielten wir $21 \cdot 72 \%$ Brom, nach $z$ weimaligem sank der Bromgehalt auf $19 \cdot 23 \%$.

Die Analyse der reinen, richtig schmelzenden Substanz ergab Folgendes:

$0 \cdot 2330 \mathrm{~g}$ Substanz gaben $0 \cdot 1015 \mathrm{~g}$ Bromsilber.

In 100 Theilen:

$$
\mathrm{Br} \ldots \ldots \ldots \underbrace{}_{18.53} \underbrace{\begin{array}{c}
\text { Berechnet } \\
\text { Gur } \mathrm{C}_{28} \mathrm{H}_{21} \mathrm{Br}
\end{array}}_{18 \cdot 30}
$$

Sämmtliche Brombestimmungen wurden infolge der schweren Zersetzlichkeit mittels der Kalkmethode ausgeführt; dem Bromderivat käme dann folgende Structurformel zu: 


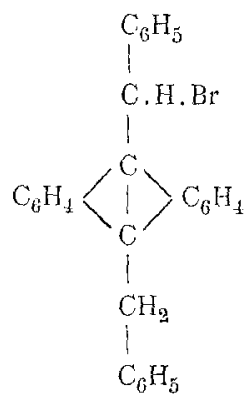

Dasselbe ist in Benzol, Schwefelkohlenstoff leicht löslich, wird dagegen von Ather in Alkohol schwer aufgenommen. Gegen chemische Agentien erweist es sich äußerst unbeständig, indem das Bromatom leicht durch andere Reste substituiert wird; selbst beim Erhitzen auf $160^{\circ}$ beginnt bereits die Abspaltung von Bromwasserstoff; das hiebei entstehende Product soll später untersucht werden.

Monooxydibenzylanthracen. Kocht man das Bromproduct circa zehn Stunden mit sehr viel Wasser bei Gegenwart von Pottasche, so spaltet sich das Brom als Bromwasserstoff ab und man erhält das Monobenzylphenylanthracarbinol:

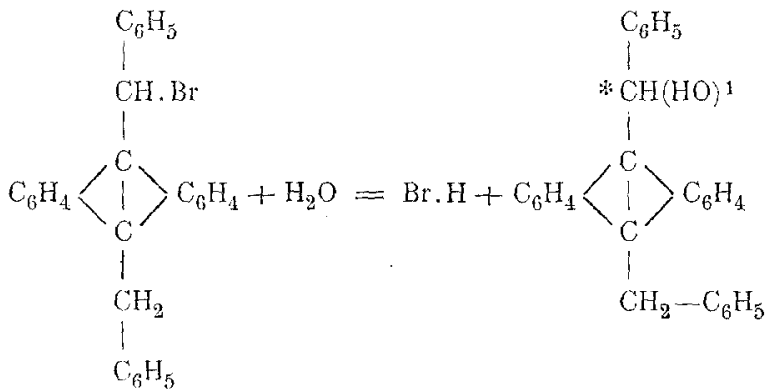

Das Auftreten von Bromwasserstoffsäure konnte mit voller Sicherheit nachgewiesen werden, das so erhaltene Product wurde abfitriert und gewaschen, erwies sich mittels der Beilstein-Probe vollständig bromfrei. Es wurde in Ather gelöst, letzterer größtentheils abdestilliert und die concentrierte Lösung

1 Da das mit * bezeichnete Kohlenwasserstoffatom als asymetrisch bezeichnet werden muss, so soll das opt. Drehungsvermögen dieser Verbindung näher untersucht werden, 
einige Zeit stehen gelassen; es scheiden sich hiebei gelbliche Krystalle aus, welche bei 225 bis $226^{\circ}$ schmelzen; dieselben waren in Äther, Benzol, Schwefelkohlenstoff leicht, dagegen in Alkohol schwer löslich. Aus letzterem Mittel krystallisieren sie in hellgelben Kryställchen, die aber viel niedriger schmelzen, weil sie durch kleinere Mengen einer in Alkohol unlöslichen Schmiere verunreinigt sind.

I. $0.134 \mathrm{~g}$ Substanz $0.4390 \mathrm{~g} \mathrm{CO}, 0.0690 \mathrm{~g} \mathrm{H}_{2} \mathrm{O}$.

II. $0.137 \mathrm{~g}$ Substanz $0.4490 \mathrm{~g} \mathrm{CO}_{2}, 0.0730 \mathrm{~g} \mathrm{H}_{2} \mathrm{O}$.

In 100 Theilen:

\begin{tabular}{|c|c|c|}
\hline \multicolumn{2}{|c|}{ Gefunden } & \multirow{2}{*}{$\underbrace{\begin{array}{r}\text { Bercchnet } \\
\text { für } \mathrm{C}_{28} \mathrm{H}_{21}\end{array}}$} \\
\hline I & II & \\
\hline $89 \cdot 37$ & $89 \cdot 54$ & $89 \cdot 83$ \\
\hline $5 \cdot 72$ & $5 \cdot 91$ & $5 \cdot 88$ \\
\hline
\end{tabular}

Interessant ist das Verhalten des Bromids gegen Eisessig: hier bildet sich beim Kochen nicht, wie zu erwarten wärc, ein Acetat, sondern, wie nachstehende Eigenschaften und Analyse zeigen, der oben beschriebene Alkohol. Das Bromid wurde mit überschüssigem Eisessig einige Zeit am Rückflusskühler bis zur klaren Lösung gekocht, dann beim Verdünnen mit Wasser scheidet sich das Product aus, welches abfiltriert, getrocknet und aus Äther umkrystallisiert, den Schmelzpunkt von 225 bis $226^{\circ}$ zeigte und der Analyse nach identisch mit dem oben erwähnten Alkohol gefunden wurde.

$0.163 \mathrm{~g}$ Substanz gaben $0.5360 \mathrm{~g} \mathrm{CO}_{2}$ und $0.0820 \mathrm{~g} \mathrm{H}_{2} \mathrm{O}$.

In 100 Theilen:

\begin{tabular}{|c|c|c|}
\hline & Gefunden & $\begin{array}{l}\text { Berechnet } \\
\text { für } \mathrm{C}_{28} \mathrm{H}_{21} \mathrm{OH}\end{array}$ \\
\hline & $89 \cdot 68$ & $89 \cdot 83$ \\
\hline & $5 \cdot 58$ & $5 \cdot 88$ \\
\hline
\end{tabular}

Diesem Thatbestand entsprechend scheint die Finwirkung des Eisessigs auf das Bromproduct merkwürdigerweise nach folgender Gleichung vor sich zu gehen: 


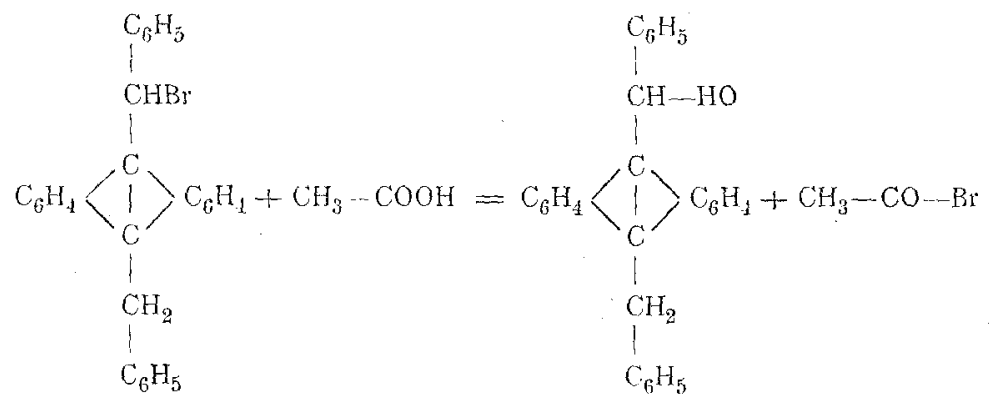

Nach Versuchen von Menschutkin sind bekanntlich die secundären Alkohole mit Eisessig erst bei $150^{\circ}$ im Druckrohr, also viel schwerer esterificierbar als die primären. Da in unserem Falle ein secundärer Alkohol vorliegt, indem die HO-Gruppe an den CH-Rest gebunden erscheint, so wäre vielleicht die Inactivität des Carbinols gegenüber dem Säurebromid durch seine Structur erklärt.

Athylester. Trägt man in das in Benzol gelöste Bromproduct etwas Alkohol ein und erwärmt ein wenig, so beginnen sich sofort weiße Blättchen auszuscheiden, deren Benzollösung stark fluoresciert. Dieselben wurden aus Benzol und Alkohol umkrystallisiert und schmelzen dann bei $218^{\circ}$ und müssen als Äthylester des vorherbeschriebenen Alkohols betrachtet werden.

I. $0.145 g$ Substanz gaben $0.4735 g \mathrm{CO}_{2}, 0.086 \mathrm{~g} \mathrm{H}_{2} \mathrm{O}$.

1I. $0.156 g$ Substanz gaben $0.5130 g \mathrm{CO}_{2}, 0.0950 g \mathrm{H}_{2} \mathrm{O}$.

In 100 Theilen:
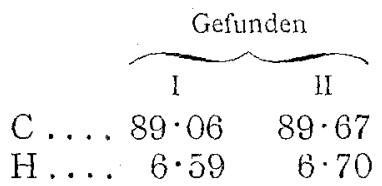

Berechnet für $\mathrm{C}_{30} \mathrm{H}_{26} \mathrm{O}$

H... 6.59

$6 \cdot 70$

$89 \cdot 55$

$6 \cdot 46$

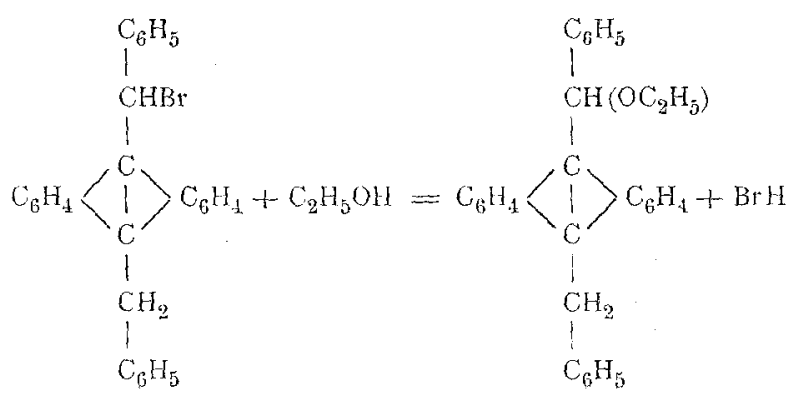


Die Fortsetzung dieser Arbeit wird hiemit vorbehalten, ebenso die Einwirkung von Benzalchlorid wie Benzotrichlorid auf Anthracen, deren Studium bereits vor einiger Zeit begonnen hat. Das in dieser Arbeit benützte blau fluorescierende Anthracen verdanken wir in ausgezeichneter Reinheit den Farbwerken vormals Meister Lucius \& Comp. in Höchst am Main, welchen wir hiefür bestens danken. 